\title{
The Web Service Modeling Toolkit - An Integrated Development Environment for Semantic Web Services (System Description)
}

\author{
Mick Kerrigan, Adrian Mocan, Martin Tanler, and Dieter Fensel \\ Digital Enterprise Research Institute (DERI), \\ Leopold-Franzens Universität Innsbruck, Austria \\ firstname.lastname@deri.org
}

\begin{abstract}
The time of engineers is a precious commodity. This is especially true for engineers of semantic descriptions, who need to be highly skilled in conceptual modeling, a skill which will be in high demand as Semantic Web technologies are adopted by industry. Within the software engineering community Integrated Development Environments (IDEs) like the Eclipse Java Development Toolkit and NetBeans have proved to increase the productivity of engineers by bringing together tools to help engineers with their everyday tasks. This paper motivates the need for such an IDE for the Semantic Web and in particular describes the Web Service Modeling Toolkit (WSMT), an Integrated Development Environment for Semantic Web Services through the WSMO paradigm.
\end{abstract}

\section{Introduction}

The combination of Semantic Web and Web service technologies, to create Semantic Web Services (SWS), with the aim of automating the Web service usage process has been the aim of the WSMO [4, WSML [5] and WSMX [] working groups over the last number of years. The research in this area has produced a conceptual model, a formal langauge and many back-end services for finding and using Web services that meet the requirements of end-users. However the process of creating the required semantic descriptions is a difficult task and the time of Ontology and Semantic Web Service engineers is a precious commodity.

Within the Semantic Web domain there is much ongoing research into how to present ontologies and semantic data to users. Each of the tools resulting from such research tend to address a given problem within the domain, with these individual research efforts rarely being aligned or integrated together. The lack of integration results in developers switching back and forward between different tools for different tasks. In this paper we introduce the Web Service Modeling Toolkit (WSMT) 1 , an Integrated Development Environment for Semantic Web Services, aimed at increasing the productivity of Ontology and Semantic Web Service engineers.

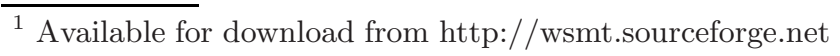


An Integrated Development Environment (IDE) is defined as a type of computer software that assists computer programmers to develop softwart 2 . The main aim of an IDE is to improve the productivity of the developer by seamlessly integrating tools for tasks like editing, file management, compilation, debugging and execution. Before the creation of the WSMT, developers of semantic descriptions using the WSMO paradigm were forced to create their ontologies, web services, goals and mediators by hand in a text editor. This has many inherent problems, as due to the lack of validation and testing support it is very easy for errors to creep into these semantic descriptions, which go unnoticed by the developer until run-time. Many other tasks that are very easy in an IDE, can be hugely time consuming without one, for example registering a semantic description with an execution environment. Providing a fully integrated suite of tools for Semantic Web Services, that meets the needs and requirements of developers should aid in the adoption of the WSMO technology and reduce the overhead of creating SWS applications.

In the following section we provide the background for our work, in section 3 we describe the problems currently addressed by the WSMT, in section 4 we describe related work and finally in section 5 we provide some conclusions and future work.

\section{Background}

The Semantic Web aims to make the vast quantities of information available on the Web machine-understandable, by the use of ontologies to annotate Web content. Web service technologies have emerged as a contender for the next generation of Web applications, essentially lifting the Web from a static collection of information to a dynamic computational entity. Web services have machineprocessable annotations that are well structured (using XML) and describe how to interface with these services. However these annotations are purely syntactic and not machine-understandable, thus large amounts of human effort is required to build Service Oriented Architectures. Semantic Web Services are the extension of ontologies to describe Web services such that a machine can reason about the functionality they provide, the mechanism to invoke them, and the data they expect as input and return as output. Once Web services are described semantically it allows for large parts of the Web service usage process to be automated. Services can be discovered based upon their functionality, can be selected based upon the quality of the service, heterogeneity issues with respect to the data they exchange or the process to invoke them can be mediated. It is important to note that the semantic descriptions to enable this functionality layer on top of the existing syntactic descriptions for existing Web services. Thus Semantic Web Services are not a reinvention of Web services but an enhancement to them. There are two main contenders in the field of Semantic Web Services, namely WSMO 4 and OWL-S[16] 15. Within the scope of this paper we focus on WSMO as the conceptual model for Semantic Web Services.

\footnotetext{
${ }^{2}$ http://en.wikipedia.org/wiki/Integrated_development_environment
} 
The Web Service Modeling Ontology (WSMO) 4] is a conceptual model for creating semantic descriptions for Web services that can be used to resolve interoperability issues and automate the Web service usage process. WSMO is based on the Web Service Modeling Framework (WSMF) [5] and as such, is based on the four main elements of the WSMF: ontologies, web services, goals and mediators. The aim of WSMO is to solve the integration problem by describing Web services semantically and by removing ambiguity about the capabilities of Web services, the problems they solve and the process of interacting with them.

The Web Service Modeling Language (WSML) 10] is a formalization of the WSMO ontology, providing a language within which the properties of Semantic Web Services can be described. There are five language variants, based on Description Logic and Logic Programming. Each language variant provides different levels of logical expressiveness [10. These variants are: WSML-Core, WSML-DL, WSML-Flight, WSML-Rule and WSML-Full. WSML-Core, which corresponds to the intersection of Description Logic and Horn Logic, provides the basis for all the variants, while WSML-Full unites the functionality of all variants. WSML Core is extended in the direction of more expressive Description Logic by WSMLDL and towards Logic Programming by WSML-Flight and WSML-Rule.

A Semantic Execution Environment (SEE) for Semantic Web Services can be used to bind service requestors and providers together at runtime using semantic descriptions of the user's goal and the provider's Web service. SEEs provide functionality for discovering, composing, selecting, mediating, and invoking Web services that match the end users requirements. There are currently two Semantic Execution Environments for WSMO, namely the Web Service Execution Environment (WSMX) [7] and IRSIII[2]. The SEE functionality is currently being standardized through the OASIS standardization.

\section{The Web Service Modeling Toolkit}

The Web Services Modeling Toolkit (WSMT) is an Integrated Development Environment (IDE) for Semantic Web Services implemented in the Eclipse framework. The WSMT aims to aid developers of Semantic Web Services through the WSMO paradigm, by providing a seamless set of tools to improve their productivity. As already mentioned a Integrated Development Environment (IDE) is defined as a type of computer software that assists computer programmers to develop software. IDE's like the Eclipse Java Development Toolkit (JDT) 3 and NetBean:4 for developing java software have proven that good tool support can improve the productivity of engineers. It could be said that the time of ontology and Semantic Web Service engineers is a more precious commodity, as the number of people who are currently skilled in conceptual modeling is much less than those that can code in Java. This underscores the need for adequate tool support for working with semantic technologies and an IDE can tie together these tools in such a way that the whole application is more than the sum of the parts

\footnotetext{
${ }^{3}$ http://www.eclipse.org/jdt/

${ }^{4}$ http://www.netbeans.org
} 
that make it up. The main advantage of using the IBM Eclipse framework is the existence of other useful plugin projects, for example the Eclipse Web Tools Platform (WTP)5, which provides tools for Web service technologies like WSDL and XML. This enables developers to put the WSMT and the WTP together to build their Web services and semantically describe them with WSMO. The process of building ontologies or describing Web services semantically involves the creation of different types of documents. The main tasks in this process revolve around four main themes:

- Editing: Firstly the actual descriptions must be created. It is important that users of different skill levels are supported within the IDE, thus editing support at different levels of abstraction should be provided. Considering ontologies, it may be more convenient for the engineer to create an ontology using a textual representation and then to use a graph based representation to learn more about the ontology.

- Validating: The most common problem that occurs when creating semantic descriptions is incorrect modeling. It can be very easy for an engineer to make a mistake without any tool support. Validation of semantic descriptions is a non trivial task and validation at both the syntactic and semantic levels can vastly reduce the time an engineer spends debugging an ontology.

- Testing: Once valid semantic descriptions exist the engineer needs to ensure that they behave in the expected manner in their intended environment prior to deploying them. Having testing integrated into the development environment reduces the overhead of the user performing a lengthy, iterative, deploy-test scenario.

- Deploying: Ultimately the descriptions created within the development environment must be used in some run-time system. Deploying descriptions can also be a huge overhead on the engineer and having tool support in an IDE can prevent mistakes occurring at this crucial stage of the process.

The WSMT focuses on three main areas of functionality, namely the engineering of WSMO descriptions, creation of mediation mappings and interfacing with execution environments and external systems. Towards these aims, the Eclipse editors and views available within the WSMT are broken up into three Eclipse perspective:6. These perspectives are the WSML, Mapping and SEE perspectives respectively and are described in the next sections.

\subsection{The WSML Perspective}

Creating ontology descriptions is a non trivial task that requires the skills of a trained ontology engineer, and many tools exist for building ontologies in OWL 16] and RDF $(\mathrm{S})[1$. In the case of WSMO the engineer must also be knowledgable about both Ontologies and Web services in order to creation semantic

\footnotetext{
${ }^{5}$ http://www.eclipse.org/webtools/

${ }^{6}$ Eclipse perspectives are used to group together editors and views that a given developer will use while performing a given set of tasks.
} 
descriptions. As already described it is important that tool support exists for efficiently using the time of this skilled engineer. Within the WSML perspective a number of tools are provided that allow users of different skill levels to create, manage and interact with semantic descriptions in the WSMO paradigm. These tools include:

- WSML Validation: WSMO4. 7 is an object model for manipulating WSMO descriptions and is capable of parsing and serializing WSML documents to and from this object model. WSMO4J also provides a validator for each of the 5 WSML variants, which is exploited within the WSMT to validate the files that are located within the WSMT workspace as the user edits these documents. This validation ensures that the engineer of the semantic descriptions gets immediate feedback of errors they create, both in the syntax and the semantics of the semantic descriptions, as they create them.

- WSML Text Editor: Until recently ontology engineers using the WSMO paradigm would create there WSMO descriptions by hand in a text editor. It is very tempting when creating a toolkit to abstract away from a text editor and to provide more advanced editing support; However in many cases the engineer is more comfortable with editing the raw text of the semantic description. This is especially true with respect to WSML, as the WSML human readable syntax is a very lightweight syntax. Within the WSMT we cater for such users and provide them with additional features including syntax highlighting, syntax completion, in line error notification, content folding and bracket highlighting.

- WSML Form based Editor: Moving up from the WSML text editor, we have abstracted to a form based editor that provides the user with an intuitive interface for building semantic descriptions by completing forms. In this editor the user can create new elements related to their semantic description and specify their properties. Within the editor the user is always manipulating a WSMO4J object model, which can be serialized to the human readable syntax by saving the editor.

- WSML Visualizer: The WSML Visualizer [8] is a fully integrated graph based visualization and editing tool for WSML. The WSML Visualizer allows the engineer to learn more information about their semantic descriptions as they create them. Normally visualization solutions are bolted on top of existing ontology engineering solutions after the fact, by providing an integrated solution the user need not switch back and forth between an editing environment and a visualizer to understand the effects of changes to the ontology. The visualizer tries to resolve the graph scalability problem that is often found with visualization solutions by breaking down the visualization into multiple levels and allowing the user to browse these levels like in a web browser. The tool includes manipulation features like zoom and rotate, along with the ability to filter nodes of a certain type.

- WSML Reasoner View: Semantic descriptions are only useful if they can eventually be reasoned over and much work is ongoing within the WSML

$\overline{7}$ http://wsmo4j.sourceforge.net/ 
community to create reasoners for all 5 variants of the WSML language. This view exposes the functionality of the WSML2Reasoner framework, which currently provides access to the Pellet 14 description logic reasoner and MINS logic programming reasonen 8 . The purpose of this view is to allow the engineer of semantic descriptions to be sure that the descriptions they create yield the expected results when put into a reasoner.

- Discovery View: When engineers are creating Semantic Web Service descriptions, it must always be at the forefront of their mind that these Web Service descriptions will ultimately be discovered by a users Goal description. The discovery view can be used in a similar way to the reasoner view to allow the engineer to check that a specified Goal matches the expected set of the Web Services in the users workspace, i.e. that the Goal will behave as expected in a given discovery engine.

\subsection{The Mapping Perspective}

When enabling interoperability between two business partners it is important that the data exchanged is correct. However it is unlikely that both partners will use the same ontology to represent their data. Ontology to ontology mediation within the WSMX environment is considered as a semi automatic process, where mappings between two ontologies are created at design time and then applied automatically at runtime in order to perform instance transformation. Automatic approaches for creating mappings do exist but their accuracy is relatively low and we believe that for business to business integration an engineer must be involved in creating and validating the mappings. This is a non trivial task and the user should be guided through the process of creating the mappings and ensuring that they are correct. Within the WSMT all mappings are in the Abstract Mapping Langauge (AML) 13] syntax, which is formalism neutral, and later grounded to WSML within WSMX.

- AML Validation: The importance of validating semantic descriptions as the user creates them was already described in section 3.1 and this is equally true for the Abstract Mapping Language. Within the WSMT we currently provide validation for the syntax of AML documents and will extend this to semantic validation when a validator for the AML semantics is available.

- AML Text Editor: The Abstract Mapping Language Text Editor provides a text editor for the human readable syntax of the AML. It features similar features to that of the WSML human readable text editor including syntax highlighting, in line error notification, content folding and bracket highlighting. This editor enables the engineer to create or modify mappings through textual descriptions, this is especially useful for tweaking mappings that have been produced by other editors.

- AML View Based Editor: The AML View Based Editor provides a graphical means to create mappings between ontologies. It is often the case that the expert that understands the problem domain and is capable of aligning

$\overline{8}$ http://tools.deri.org/mins/ 
the two ontologies is not also a specialist in logics. The suggestion of possible mappings is done by using a set of algorithms for both lexical and structural analysis of the concepts. Additionally, the guidance is offered by decomposition and context updates. As described in [11, the graphical point of view adopted to visualize the source and target ontologies makes it easier to identify certain types of mismatches. This viewpoints are called perspectives and it is argued that by using combinations of these perspectives on the source and target ontologies, certain types of mappings can be created using only one simple operation, map, combined with mechanisms for ontology traversal and contextualized visualization strategies.

- AML Mapping Views: The AML Mapping Views have the role of providing a light overview on the mappings created either by using the AML Text Editor or the AML View Based Editor. Instead of seeing the full description of mappings it is also useful to see a more condensed version of this information with the entities in the source and in the target that are mapped and the conditions associated with them. For this purpose there been four types of Eclipse views defined (Concept2Concept, Attribute2Attribute, Concept2Attribute and Attribute2Concept), each corresponding to the combinations of the entities that can participate in a mapping.

- MUnit Testing View: Mappings must be updated as ontologies evolve, it can be hard for the engineer to be aware of the effects that these constant changes have. The MUnit unit testing view for the Abstract Mapping Language gives the engineer support to ensure that instances are correctly transformed. The user can define pairs of sources and targets, specifying that the result of transforming the sources, using the existing mappings, should be the targets. These tests can then be incrementally run by the engineer when validation of the mappings is required.

\subsection{The SEE Perspective}

Ultimately the purpose of creating semantic descriptions in WSMO and defining mappings between ontologies is to allow automation of the Web service usage process and to bind requester and provider at runtime with minimal human intervention. As described in section 2, Semantic Execution Environments, like WSMX and IRSIII provide automatic discovery, composition, selection, mediation, and invocation of Semantic Web Services. Many of the tasks performed by the Semantic Web Service engineer involve interfacing with these SEEs. Functionality is provided within the WSMT to reduce the effort normally spent interacting with these environments.

- Integration with WSMX: WSMX exposes a number of Web service interfaces that give access to its functions. These Web services can be split into two categories, namely services related to executing WSMX and services related to storing and retrieving descriptions. The SEE Perspective provides access to both of these types of services, users can right click on WSML 
and AML documents in the workspace and use the descriptions contained within these documents to invoke the entry points of WSMX i.e. AchieveGoal, InvokeWebService or these descriptions can be stored to the internal repositories of WSMX. The SEE perspective also shows all the descriptions currently stored within the WSMX repositories and allows the user to download these descriptions into their workspace.

- Integration with IRSIII: Similar functionality as for the WSMX system is available for the IRSIII system. One interesting difference is that all data within IRSIII is described in terms of the Open Universities OCML 12 format, and the WSMT must translate back and forth from this format when sending data to or retrieving data from IRSIII.

\section{Related Work}

This section outlines other efforts in the direction of creating integrated environments aiding users of semantic technologies. For this we have selected the most popular tool for creating ontologies in OWL, namely the Protégé OWL Plugin, along with WSMO Studio, which is the closest alternative to the WSMT for creating WSMO descriptions.

Protégé [6] is a free open source ontology engineering environment that can be extended to support different ontology formats. Through a collection of these extensions Protégé currently supports Frames, XML Schema, RDF $(\mathrm{S})$ and OWL. The Protégé OWL Plugin [9] was developed at Stanford Medical Informatic 9 as a tool for editing the Web Ontology Language (OWL) [16]. The plugin attempts to abstract the user away from the underlying OWL RDF syntax and provides a number of graphical widgets that can be accessed by the user for building ontologies and creating instance data. The Protégé OWL Plugin can also be extended with plugins, existing plugins provide functionality for visualizing OWL ontologies, integrating with OWL reasoners etc. The main limitation of Protégé is that, while it is the standard toolkit used within the Semantic Web community for building ontologies, other tools are not integrated with this environment. Semantics will soon be present in many different parts of computer science and as already described integrating Semantic Web tools into the Eclipse framework allows for them to be used side by side with other toolkits like the Java Development Toolkit (JDT) or Web Tools Platform (WTP).

WSMO Studio 3 is a collection of tools for editing WSMO descriptions developed for the Eclipse Framework by OntoText Labs10, Sirma. WSMO Studio offers the engineer functionality for editing WSMO descriptions through the WSML paradigm. The main difference between the WSMT and WSMO Studio is the level of abstraction. WSMO Studio focuses squarely on editing descriptions at the form based level and provides only basic functionality in a text editor for WSML, i.e. Syntax highlighting and no ontology visualization functionality.

\footnotetext{
${ }^{9}$ http://smi.stanford.edu/

${ }^{10}$ http://www.ontotext.com
} 
With respect to Semantic Execution Environments, WSMO Studio has functionality for invoking the IRSIII system through its repositories view but does not provide any functionality for integrating with WSMX. The choice of Eclipse by both environments allows for the tools of each, which in many ways complement each other, to be placed in the same application.

\section{Conclusions and Future Work}

This paper has described the need for an Integrated Development Environment for the Semantic Web and for Semantic Web Services. It has also introduced the Web Service Modeling Toolkit (WSMT), which aims to provide such an IDE to improve the productivity of engineers of semantic descriptions. Section 3 introduced the concepts of editing, validating, testing and deploying in an IDE. The following table shows how the Web Service Modeling Toolkit meets these four concepts for each of the descriptions that it supports:

\begin{tabular}{lll}
\hline Feature & Ontology, Web Service \& Goal & Mappings \\
\hline Editing & Text, Form \& Graph & Text \& Dual Tree \\
Validating & Syntactic \& Semantic & Syntactic \\
Testing & Reasoning View \& Discovery View & MUnit View \\
Deploying & SEE Perspective & SEE Perspective \\
\hline
\end{tabular}

Future work in the WSMT includes automation of the process of ensuring that semantic description behave as expected, by the addition of unit testing functionality for WSML. This unit testing functionality will allow the user to ensure that their ontologies behave in the expected way as they evolve. Further integration with the WSMX and IRSIII systems will also be added to the SEE perspective, allowing the user to visualize the processes that are executing within the Semantic Execution Environments. Thus allowing engineers to debug their descriptions with respect to their behavior within a SEE.

\section{Acknowledgments}

The work is funded by the European Commission under the projects ASG, DIP, enIRaF, InfraWebs, Knowledge Web, Musing, Salero, SEKT, SEEMP, SemanticGOV, Super, SWING and TripCom; by Science Foundation Ireland under the DERI-Lion Grant No.SFI/02/CE1/I13 ; by the FFG (Österreichische Forschungsförderungsgeselleschaft $\mathrm{mbH}$ ) under the projects Grisino, RW ${ }^{2}$, SemNetMan, SeNSE, TSC and OnTourism. 


\section{References}

1. D. Brickley and R.V. Guha. Resource Description Framework (RDF) Schema Specification 1.0, W3C Recommentdation, 2000.

2. L. Cabral, J. Domingue, S. Galizia, A. Gugliotta, B. Norton, V. Tanasescu, and C. Pedrinaci. IRS-III: A Broker for Semantic Web Services Based Applications. In Proc. of the 5th Int'l Semantic Web Conf (ISWC), 2006.

3. M. Dimitrov, A. Simov, V. Momtchev, and D. Ognyanov. WSMO Studio - an Integrated Service Environment for WSMO. In WSMO Implementation Workshop 2005, volume 134 of CEUR Workshop, 2005.

4. C. Feier, A. Polleres, D. Roman, J. Domingue, M. Stollberg, and D. Fensel. Towards Intelligent Web Services: The Web Service Modeling Ontology (WSMO). In Proc. of the Int'l Conf on Intelligent Computing (ICIC), 2005.

5. D. Fensel and C. Bussler. The Web Service Modeling Framework (WSMF). Electronic Commerce Research and Applications, 1(2):113-137, 2002.

6. J. H. Gennari, M. A. Musen, R. W. Fergerson, W. E. Grosso, M. Crubzy, H. Eriksson, N. F. Noy, and S. W. Tu. The Evolution of Protégé: An Environment for Knowledge-Based Systems Development. Technical report, 2002.

7. A. Haller, E. Cimpian, A. Mocan, E. Oren, and C. Bussler. WSMX - A Semantic Service-Oriented Architecture. In Proc. of the Int'l Conf on Web Services (ICWS), 2005.

8. M. Kerrigan. WSMOViz: An Ontology Visualization Approach for WSMO. In Proc. of the 10th Int'l Conf on Information Visualization, 2006.

9. H. Knublauch, R. W. Fergerson, N. F. Noy, and M. A. Musen. The Protégé OWL Plugin: An Open Development Environment for Semantic Web Applications. In Proc. of the 3rd Int'l Semantic Web Conf (ISWC), 2004.

10. H. Lausen, J. de Bruijn, A. Polleres, and D. Fensel. WSML - A Language Framework for Semantic Web Services. In Proc. of the W3C Workshop on Rule Languages for Interoperability, 2005.

11. A. Mocan, E. Cimpian, and M. Kerrigan. Formal Model for Ontology Mapping Creation. In Proc of the 5th Int'l Semantic Web Conf (ISWC 2006), 2006.

12. E. Motta. Reusable Components for Knowledge Modelling. Case Studies in Parametric Design Problem Solving, volume 53 of Frontiers in Artificial Intelligence and Applications. IOS Press, 1999.

13. F. Scharffe and J. de Bruijn. A language to specify mappings between ontologies. In IEEE Conference on Internet-Based Systems SITIS6, 2005.

14. E. Sirin, B. Parsia, B. Cuenca Grau, A. Kalyanpur, and Y. Katz. Pellet: A Practical OWL-DL Reasoner. Submitted for publication at "Journal of Web Semantics", 2006. Available from: http://www.mindswap.org/papers/PelletJWS.pdf.

15. N. Srinivasan, M. Paolucci, and K. Sycara. Adding OWL-S to UDDI, Implementation and Throughput. In Proc. of 1st Int'l Workshop on Semantic Web Services and Web Process Composition (SWSWPC), 2004.

16. W3C. OWL Web Ontology Language Reference. Technical report, 2004. 